\title{
Bootstrapping an End-to-End Natural Language Interface for Databases
}

\author{
Nathaniel Weir \\ Brown University \\ Providence, USA \\ nathaniel_weir@brown.edu
}

ACM Reference Format:

Nathaniel Weir and Prasetya Utama. 2019. Bootstrapping an End-toEnd Natural Language Interface for Databases. In 2019 International Conference on Management of Data (SIGMOD '19), June 30-fuly 5, 2019, Amsterdam, Netherlands. ACM, New York, NY, USA, 3 pages. https://doi.org/10.1145/3299869.3300105

\section{PROBLEM AND MOTIVATION}

Structured query language (SQL), despite its expressiveness, may hinder users with little or no relational database knowledge from exploring and making use of the data stored in an RDBMS. In order to effectively leverage their data sets, users are required to have prior knowledge about the schema of their database, such as table names, columns and relations, as well as a working understanding of the syntax and semantics of SQL. These requirements set a high bar for entry for democratized data exploration and thus have triggered new efforts to develop alternative interfaces that allow non-technical users to explore and interact with their data conveniently and effectively.

Recent efforts have used deep learning models to create Natural Language Interfaces to Databases (NLIDBs) [4, 11, 12], leveraging Bi-LSTM translation models to parse natural language utterances and synthesize their corresponding SQL query over a given database. These approaches have achieved state-of-the art performance on all nl-to-sql (nl2sql) benchmarks, but they rely on a supervised learning framework that requires substantial training data from the target domain. This training data is assumed to have been acquired a priori and crafted to be well-representative of the types of queries one might ask in the target domain. This assumption poses a major problem for systems geared toward accessibility for nontechnical users. It is improper to assume that

Permission to make digital or hard copies of part or all of this work for personal or classroom use is granted without fee provided that copies are not made or distributed for profit or commercial advantage and that copies bear this notice and the full citation on the first page. Copyrights for thirdparty components of this work must be honored. For all other uses, contact the owner/author(s).

SIGMOD '19, June 30-fuly 5, 2019, Amsterdam, Netherlands

(c) 2019 Copyright held by the owner/author(s).

ACM ISBN 978-1-4503-5643-5/19/06.

https://doi.org/10.1145/3299869.3300105

\author{
Prasetya Utama \\ TU Darmstadt \\ Darmstadt, Germany \\ utama@aiphes.tu-darmstadt.de
}

users without knowledge of databases or SQL will be able to craft sufficient training data for any new database that they want to explore.

In this work, we introduce a system that addresses these training data issues by leveraging a synthetic data generator that bootstraps the learning process to produce robust and effective models in any new domain.

\section{BACKGROUND AND RELATED WORK}

The task of synthesizing an SQL program from natural language text has been studied within both the NLP and database research communities since the late 1970s [7, 15].

In the database community, notable NLIDBs such as NaLIR [6] define a rule-based system based on the syntactic structure of the NL utterance (i.e. using part-of-speech tags [9] or dependency relations [6]). Others such as [10] rely on heavy manual overhead for each new database to be handled. While some of these approaches require minimal manual overhead, they are inherently limited in terms of linguistic coverage and, as we show in evaluation, break down when given queries with simple lexical or syntactic changes.

Within the NLP community, much success has been found in applying bi-LSTM sequence-to-sequence (seq2seq) neural networks [1, 2, 4]; these models have consistently outperformed their statistical predecessors. However, few have addressed the aforementioned training data bottleneck when using these complex networks [11] .

[13] introduce SyntaxSQLNet, a cross-domain translation model that operates over multiple databases by taking as input a target dataset's table and column names. This opens up the opportunity to leverage training data from many domains instead of a single one. Their system sets state-ofthe-art performance on Spider [14], a text-to-SQL benchmark containing queries over 206 different databases. Like ours, their training pipeline leverages synthetic data generation. Our approach differs from theirs in that they use a far less extensive set of query templates that only cover single-table operations, while ours cover multi-table join queries. Their set of query templates is also not geared towards boosting linguistic robustness and has a very limited variety of NL queries that can be generated for each SQL pattern. It is also important to note that their generation process serves 


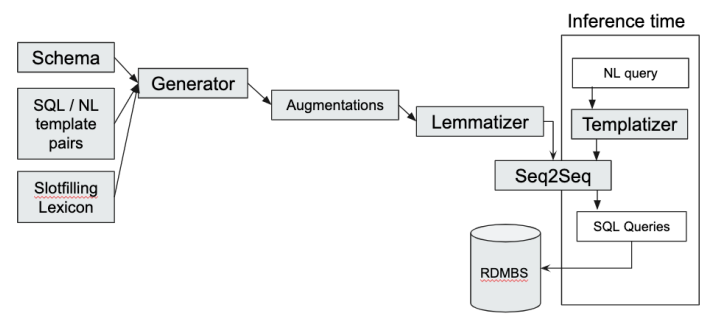

Figure 1: DBPal System Overview

solely as a training set augmentation, while ours can serve either as augmentation or as standalone training data to train zero-shot models in new domains.

\section{APPROACH AND UNIQUENESS}

The main contribution of this work addresses the training data bottleneck from a novel angle. We introduce a bootstrapping approach for training a neural NLIDB on any new database schema. Using a set of over 100 domain-independent seed templates, our system generates vast amounts of domainspecific training examples of natural language utterances with their corresponding SQL queries. Each SQL pattern in the grammar of templates is matched with many distinct NL patterns. The NL and SQL templates contain slots to be filled in by database elements, while the NL templates also contain phrase slots to be filled in using a back-end English lexicon. The set of SQL patterns covered includes aggregations, simple nested queries and column joins.

We further add variety to the training data through a number of augmentation steps that draw inspiration from various works in computer vision and machine translation, such as random word dropout and automatic paraphrasing. The produced training data leads to a trained model that is more robust to linguistic variation than its predecessors.

We present our translation model training framework as a working end-to-end system called DBPal, which requires minimal manual annotation effort by a user who is not wellversed in SQL-it only requires schema elements to be paired with simple natural language utterances. An overview of our full pipeline architecture is illustrated in Figure 1.

Our approach uniquely expands upon the typical supervised learning framework of previous models. We argue that our use of extensive linguistically-aware templates to bootstrap training provides a comparable breadth of coverage as that of manually collected training data. Furthermore, we argue that our techniques for augmenting the generated data further increase the linguistic robustness and domain adaptability of any model trained wholly or partially on it.

\section{RESULTS AND CONTRIBUTIONS}

We first show the result of generating and training on synthetic training data for a single target database. We use two

\begin{tabular}{|l|l|l|}
\hline & Patients & GeoQuery \\
\hline NaLIR & $15.60 \%$ & $7.14 \%$ \\
Supervised seq2seq & N/A & $\mathbf{8 3 . 9} \%$ \\
DBPal & $\mathbf{7 5 . 9 3 \%}$ & $55.40 \%$ \\
\hline
\end{tabular}

Table 1: Accuracy comparison between DBPal and other baselines on the two benchmark datasets

\begin{tabular}{|l|lllll|}
\hline query difficulty & easy & med & hard & extra & all \\
\hline count & 250 & 440 & 174 & 170 & $\mathbf{1 0 3 4}$ \\
\hline Base & .360 & .157 & .195 & .041 & $\mathbf{. 1 9 3}$ \\
No-Manual & .137 & .075 & 0 & 0 & $\mathbf{. 0 6 8}$ \\
Train-Synthetic & .404 & .252 & .184 & .018 &. $\mathbf{2 3 9}$ \\
All-Synthetic & .460 & .273 & .264 & .065 & $\mathbf{. 2 8 2}$ \\
\hline
\end{tabular}

Table 2: Translation Accuracy for SyntaxSQLNet with DBPal Training Augmentations

databases: GeoQuery, a well-known semantic parsing benchmark adapted nl-to-sql task ([8], [3], [4]), and the Patient dataset, a benchmark we crafted to measure the linguistic robustness of translation systems when asked to perform simple, single-table queries of a hospital database.

For GeoQuery, we replicate the work of [4] and show the result of replacing their model's manually-annotated training data with synthetic data generated by DBPal. For both benchmarks, we also evaluate against NaLIR [5], a statistical translation system which, upon its introduction, was considered the state-of-the-art NLIDB.

Results are shown in Table 1 . The model trained on only synthetic queries-thus not requiring any user overheadvastly outperforms the rule-based system and performs comparably to the supervised version whose training data closely resembles the test queries.

We then show the benefits of using our synthetic data generation on a cross-domain model that is trained to translate queries over multiple databases. We augment the training data of SyntaxSQLNet [13], the model with state-of-the-art performance on the Spider benchmark [14]. We present two versions of augmentation: first by adding synthetic queries over the databases in the training set, and second by adding queries over databases in both the train and target sets, thus mimicking the scenario where target database schemas are known, but no training examples are available. Results are shown in Table 2 . The addition of synthetic data over the training databases increases accuracy incrementally, while adding synthetic data over the target datasets increases it further.

The results of our work show that one can build an end-toend NLIDB that does not require the high manual overhead of training data curation and performs competitively with existing approaches that do. Furthermore, our approach to bootstrapping the training pipeline is model-agnostic and extensible; it can be used to increase linguistic robustness and bootstrap the training of translation models with differing architectures and purposes. 


\section{REFERENCES}

[1] R. Cai, B. Xu, Z. Zhang, X. Yang, Z. Li, and Z. Liang. An encoderdecoder framework translating natural language to database queries. In $I F C A I, 2018$.

[2] C. Finegan-Dollak, J. K. Kummerfeld, X. Lin, K. Ramanathan, S. Sadasivam, R. Zhang, and D. R. Radev. Improving text-to-sql evaluation methodology. CoRR, abs/1806.09029, 2018

[3] A. Giordani and A. Moschitti. Translating questions to sql queries with generative parsers discriminatively reranked. In COLING, 2012.

[4] S. Iyer, I. Konstas, A. Cheung, J. Krishnamurthy, and L. Zettlemoyer Learning a neural semantic parser from user feedback. In Proceedings of the 55th Annual Meeting of the Association for Computational Linguistics, ACL 2017, Vancouver, Canada, July 30 - August 4, Volume 1: Long Papers, pages 963-973, 2017.

[5] F. Li et al. Nalir: an interactive natural language interface for querying relational databases. In SIGMOD, pages 709-712, 2014.

[6] F. Li and H. V. Jagadish. Constructing an interactive natural language interface for relational databases. PVLDB, 8:73-84, 2014.

[7] A.-M. Popescu, A. Armanasu, O. Etzioni, D. Ko, and A. Yates. Modern natural language interfaces to databases: Composing statistical parsing with semantic tractability. In Proceedings of the 20th international conference on Computational Linguistics, page 141. Association for Computational Linguistics, 2004.
[8] A.-M. Popescu, O. Etzioni, and H. A. Kautz. Towards a theory of natural language interfaces to databases. In IUI, 2003.

[9] R. A. P. Rangel, J. P. Ortega, J. J. G. Barbosa, A. F. Gelbukh, G. Sidorov, and M. MyriamJ.Rodríguez. A domain independent natural language interface to databases capable of processing complex queries. In MICAI, 2005.

[10] D. Saha, A. Floratou, K. Sankaranarayanan, U. F. Minhas, A. R. Mittal, and F. Özcan. Athena: An ontology-driven system for natural language querying over relational data stores. Proc. VLDB Endow., 9(12):12091220, Aug. 2016.

[11] Y. Wang, J. Berant, and P. Liang. Building a semantic parser overnight. In ACL, 2015.

[12] X. Xu, C. Liu, and D. Song. Sqlnet: Generating structured queries from natural language without reinforcement learning. CoRR, abs/1711.04436, 2017.

[13] T. Yu, M. Yasunaga, K. Yang, R. Zhang, D. Wang, Z. Li, and D. R. Radev. Syntaxsqlnet: Syntax tree networks for complex and cross-domaintextto-sql task. In EMNLP, 2018.

[14] T. Yu, R. Zhang, K. Yang, M. Yasunaga, D. Wang, Z. Li, J. Ma, I. Li, Q. Yao, S. Roman, Z. Zhang, and D. Radev. Spider: A large-scale human-labeled dataset for complex and cross-domain semantic parsing and text-to-sql task. In EMNLP, 2018.

[15] J. M. Zelle and R. J. Mooney. Learning to parse database queries using inductive logic programming. In AAAI/IAAI, Vol. 2, 1996. 